\title{
Determinantes del Marketing Boca a Boca en el Contexto Universitario: El Rol de la Satisfacción y la Lealtad de los Estudiantes
}

\author{
Iván A. Veas-González ${ }^{1,2^{*}}$, Aurora D. Sanchez-Ortiz ${ }^{1}$ y Carmen Perez-Cabañero². \\ (1) Universidad Católica del Norte, Facultad de Economía y Administración, Depto. de Administración, \\ Avda. Angamos 0610, Antofagasta-Chile (e-mail: iveas@ucn.cl; asanchez@ucn.cl) \\ (2) Universidad de Valencia, Facultad de Economía, Depto. de Comercialización e Investigación de \\ Mercados, Av. de los Naranjos, S/N, 46022, Valencia-España. (e-mail: carmen.perez-cabanero@uv.es)
}

Recibido May. 11, 2018; Aceptado Jul. 18, 2018; Versión final Sep. 12, 2018, Publicado Feb. 2019

\section{Resumen}

Este artículo analiza los antecedentes del Marketing Boca a Boca en el contexto de instituciones de educación superior en Chile. El modelo de investigación propone como antecedentes del Marketing Boca a Boca la satisfacción y la lealtad de los estudiantes. Establece también que la variable satisfacción de los estudiantes es influenciada por las variables imagen y valor percibido de la Universidad. A partir de una muestra de 317 estudiantes y aplicando SMARTPLS 3.0, se confirmaron todas las relaciones propuestas en el modelo, siendo la satisfacción el principal antecedente en este contexto de Marketing Boca a Boca.

Palabras clave: marketing boca a boca; satisfacción; imagen; valor percibido; lealtad

\section{Word of Mouth Determinants in the University Context: The Role of Satisfaction and Student Loyalty.}

\begin{abstract}
This article analyzes the characteristics of Word of Mouth marketing (WOM) in the context of higher education institutions in Chile, particularly students' satisfaction and loyalty. Likewise, the model analyzes how the image of the University and the perceived value influence on students' satisfaction. From a sample of 317 students and applying SMARTPLS 3.0, all the proposed relationships were confirmed, being satisfaction, the main characteristics considered in this context of WOM marketing.
\end{abstract}

Keywords: word of mouth; satisfaction; image; perceived value; loyalty 


\section{INTRODUCCIÓN}

Durante las últimas décadas las instituciones de educación superior se han visto enfrentadas a un mercado altamente competitivo en la mayoría de los países, pues compiten por atraer a los mejores estudiantes en sus procesos de admisión (Luque y del Barrio, 2007; Harrisson-Walker, 2010). Dichas instituciones se enfrentan también a una creciente competencia debido a la globalización, las universidades compiten con otras semejantes en su propio país y fuera del país, siendo cada vez más conscientes de su posición en diversos rankings nacionales e internacionales (Díaz-Méndez y Gummesson, 2012). En este entorno, las instituciones de educación superior públicas y privadas tienen que adaptarse a las demandas de sus clientes potenciales, que son estudiantes cada vez más exigentes y conocedores de la oferta mundial.

Actualmente en Chile, el mercado de la educación superior también se encuentra en una creciente competencia, tanto por la captación de alumnos, como de recursos y reputación (Brunner et al., 2005). Muchos directivos de instituciones de estudios superiores están preocupados por aumentar el número de nuevos estudiantes y por mantener a los estudiantes actuales fidelizados a sus modelos educativos (Fielden et al., 1993). Es por ello que el análisis de los factores que pueden influir en la elección de una universidad es crucial para los gestores de estas instituciones. El modelo propuesto analiza como antecedentes del Marketing Boca a Boca la satisfacción y la lealtad de los estudiantes universitarios en el contexto de Chile. El modelo incluye también la imagen de la universidad y el valor percibido como antecedentes de la satisfacción de los estudiantes universitarios. Respecto a la estructura de este trabajo, tras revisar la literatura y formular las hipótesis asociadas al modelo propuesto, se describe la metodología y los principales resultados alcanzados. Finalmente se expresan las conclusiones, y recomendaciones para los gestores de instituciones de educación superior.

\section{REVISIÓN DE LA LITERATURA Y FORMULACIÓN DE HIPÓTESIS}

En este trabajo proponemos como variable crucial en el contexto de la educación superior el Marketing Boca a Boca. El Marketing boca a boca es la comunicación acerca de la evaluación de bienes y servicios entre personas que son independientes de la compañía que les proporciona el bien o servicio y en un medio que se percibe como independiente de la empresa (Silverman, 2001). El Marketing Boca a Boca procede principalmente de los llamados grupos de referencia y pertenencia, esto es, los grupos formados por amigos y miembros de la familia que ejercen influencia sobre la decisión del estudiante. Algunos modelos que tratan de predecir el comportamiento del consumidor, se basan esencialmente en dos factores: el resultado predictivo del acto del consumidor y en cómo las opiniones de su grupo de referencia pueden afectar su actuar (Jagdish et al., 1994). Harrison-Walker (2001) señalan que el Marketing Boca a Boca es la comunicación informal persona a persona, que se genera entre un comunicador no comercial y un receptor, basada en la percepción y/o experiencia del primero, con respecto a una determinada marca, un producto, una organización o algún determinado servicio. Como el Marketing Boca a Boca es una herramienta de bajo costo y representa una forma fiable de transmisión de información sobre productos y servicios, juega un papel trascendental en la difusión de información de los mercados de consumo y en la formación de las actitudes tanto en consumidores como en personas con potencial para serlo (Brown y Reingen, 1987; Mourali et al., 2005).

En el modelo propuesto, además de la variable Marketing Boca a Boca, se incluyen otras variables relacionadas que se explican a continuación. En primer lugar, incluimos en el modelo la Imagen de la universidad. Hoy en día el concepto de Imagen Corporativa se puede extender al contexto universitario o incluso al de una determinada escuela o facultad (Maric., et al, 2010), pero dado la multiplicidad de definiciones se hace importante poder determinar lo que se entenderá para este estudio por imagen de la universidad y los elementos con los que se relaciona dicho concepto. Kazoleas, et al. (2001) señalaron que la imagen de una institución se genera en el público y es el resultado de la interpretación que realiza éste de la información o desinformación sobre la organización. Helegsen y Nesset, (2007), argumentan que una imagen es la impresión general que se manifiesta en la mente de los consumidores o clientes acerca de una determinada firma En el contexto de la educación superior la imagen de la universidad tiene una influencia sobre la satisfacción de los estudiantes (Beerli et al., 2002; Schlesinger et al., 2017). En base a esto, se propone la siguiente hipótesis:

H1: La imagen de la universidad tiene una influencia directa y positiva sobre la satisfacción de los estudiantes. En segundo lugar incluimos el Valor percibido. De acuerdo con Zeithaml (1988), el valor percibido es la evaluación global que tiene un cliente de la utilidad de un producto o servicio, basado en la percepción de los beneficios recibidos versus los sacrificios realizados. Por su parte, Martín et al (2004), señala que el valor percibido de los servicios, es el juicio que realiza el cliente sobre el servicio que recibe, en donde todos los beneficios y sacrificios son percibidos a la vez y procesados en la mente del cliente, lo que conduce a una evaluación global. El valor representa entonces una compensación entre los componentes más importantes que son entregados a cambio de los que es recibido (Alves, 2011) 
En el ámbito de la educación se ha demostrado que el valor percibido tiene una influencia directa sobre la satisfacción de los alumnos (Hartman y Schmidt ,1995; Webb y Jagun, 1997; Alves y Raposo, 2007). Teniendo en consideración lo anteriormente señalado se puede determinar la siguiente hipótesis:

H2: El valor percibido sobre la universidad tiene un efecto directo y positivo sobre la satisfacción de los estudiantes.

En tercer lugar analizamos la satisfacción como antecedente del Marketing Boca a Boca. La literatura señala que la satisfacción de los estudiantes es el elemento central de reclutamiento y retención de los mismos al interior de las instituciones de educación superior (Rogers y Smith, 2011). Según Oliver (1980), la satisfacción resulta de la comparación y evaluación del desempeño de productos o servicios, con las expectativas que se poseen de los mismos antes del acto de compra o consumo. Es decir, se considera como un juicio evaluativo respecto de una post-elección relativo a una decisión de compra específica (Oliver y DeSarbo, 1988). Cabe añadir que la satisfacción del consumidor está asociada a un componente afectivo que resulta de la evaluación global de todos los aspectos que conforman una relación (Sanzo et al., 2003).

El nivel de satisfacción del cliente afecta a dos tipos de comportamiento de compra: el deseo de recompra y el Marketing Boca a Boca (Ranaweera y Prabhu, 2003). La probabilidad de que los clientes generen Marketing Boca a Boca depende del producto y de los proveedores de servicios. Primero, depende del nivel en el que el rendimiento del producto o servicios excede de las expectativas de los clientes (Maxham y Netemeyer, 2002). En segundo lugar, depende del nivel en el que no se cumplen las expectativas de los clientes; una experiencia desagradable tiene como repercusión el boca a boca negativo, expresando sentimientos negativos como la ira, para reducir su ansiedad y advertir a los demás (Sweeney et al., 2008). Investigadores como Brown et al. (2005), Heitmann et al. (2007) y Wangenheim y Bayón (2007) creen que la satisfacción de un cliente con su proveedor de productos o servicios afecta significativamente en la aparición del boca a boca.

De Matos y Rossi (2008), señalan que los altos niveles de satisfacción son un antecedente del marketing boca a boca positivo. Según Schertzer y Schertzer (2004), en el ámbito de la educación los estudiantes satisfechos pueden atraer a nuevos alumnos por la comunicación oral positiva. Por lo que, si una universidad es capaz de generar satisfacción en sus estudiantes a través de la oferta o la entrega de servicios que la misma realiza, se debe obtener un resultado beneficioso. Este resultado se centra en la comunicación interpersonal positiva de sus estudiantes con otros estudiantes potenciales o en su entorno inmediato (Marzo et al., 2005b). Teniendo en consideración lo anteriormente señalado se puede determinar la siguiente hipótesis:

H3: La satisfacción de los estudiantes tiene una influencia directa y positiva sobre la intención del Marketing Boca a Boca

Además señalamos en el modelo propuesto la relación entre satisfacción y lealtad. Los clientes satisfechos están más motivados a seguir construyendo relaciones positivas con los proveedores (Selnes, 1998). De forma similar, pero en el contexto de la educación superior, la satisfacción, está positivamente relacionada con la lealtad de los estudiantes (Alves y Raposo, 2010; Schlesinger et al., 2014, Cabana et al., 2016). De lo que se puede determinar lo siguiente:

H4: La satisfacción de los estudiantes tiene una influencia directa y positiva sobre la lealtad.

Finalmente incluimos como antecedente del Marketing Boca a Boca la lealtad. La lealtad es una actitud positiva hacia la marca y la promesa, por parte del consumidor, de generar la recompra de un determinado producto o servicio, sin afectarle los esfuerzos de marketing puestos en marcha por el resto de las empresas para lograr el cambio (Oliver, 1999). Por lo tanto, la lealtad del cliente puede ser visto como un concepto que contiene un componente actitudinal tripartito (cognitiva, afectiva y conativa) y un componente conductual estrechamente relacionados; este último guarda relación con repetir la acción de patrocinio por parte del cliente. (Lam et al., 2004). La lealtad del estudiante, sin embargo, no se limita al período durante el cual los estudiantes están formalmente registrados como estudiantes, sino que mucho más allá de la titulación de este (Marzo et al, 2005a). Según Chen (2016), cuando los estudiantes tienen mayor lealtad con sus universidades, se mejora su experiencia satisfactoria y se influye positivamente en las recomendaciones hacia los demás, es decir, que una mayor lealtad mejora su intención de Boca a Boca. Por lo anteriormente señalado, se puede determinar la siguiente hipótesis:

H5: La lealtad de los estudiantes tiene una influencia directa y positiva sobre la intención del Marketing Boca a Boca.

El modelo propuesto se ilustra en la figura 1 siguiente. 


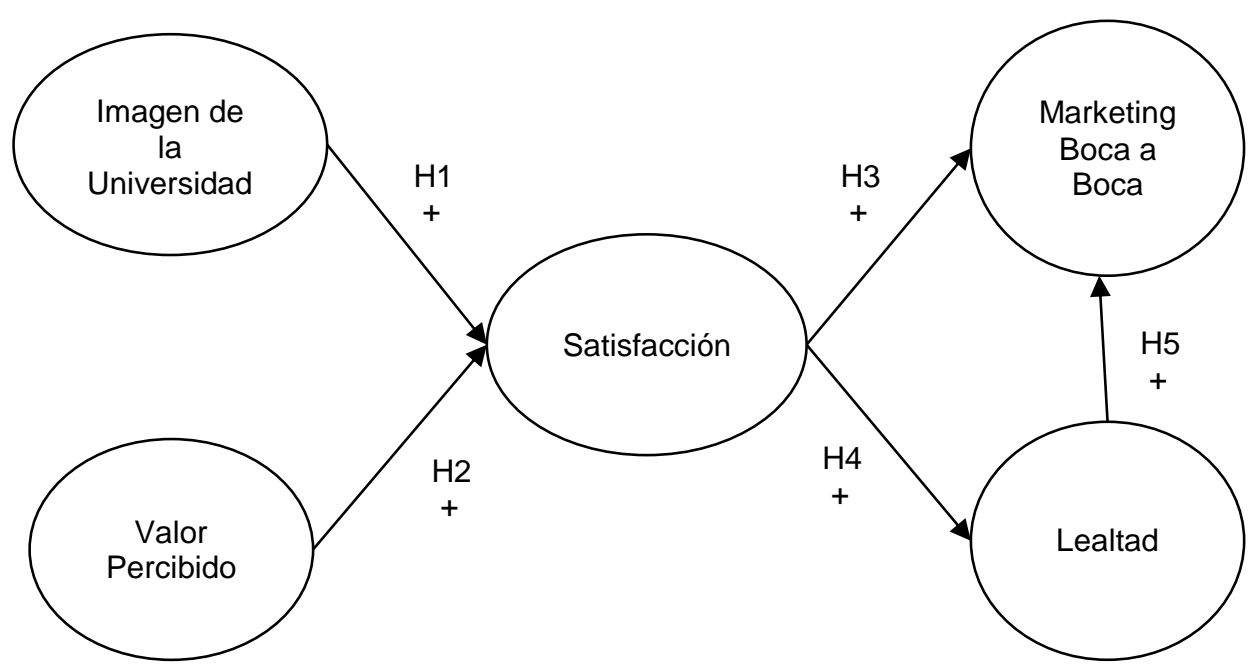

Fig. 1. Modelo Propuesto a partir de la base conceptual analizada.

\section{METODOLOGÍA}

Con el propósito de validar empíricamente las hipótesis planteadas en el estudio, se diseñó una encuesta estructurada en base a instrumentos de medida obtenidos de la revisión de la literatura (ver Tabla 1). Dichos instrumentos fueron adaptados al español y al contexto de estudio. Se realizó una prueba piloto con 50 sujetos para comprobar esta adaptación. Finalmente, la encuesta incluyó 26 preguntas de las cuales 7 estaban orientadas a obtener características sociodemográficas y 19 a la medición de cada uno de las variables incluidas en el modelo. Se utilizó una escala de Likert de 7 puntos, donde 1 representa a la respuesta "Totalmente en Desacuerdo" y 7 "Totalmente de Acuerdo" Se envió la encuesta definitiva a 1000 sujetos previamente contactados pertenecientes a dos Universidades en Chile, particularmente de la ciudad de Antofagasta. Se recibieron 317 respuestas durante los meses de Junio, Julio y Agosto del año 2015. La evaluación y validez del modelo estructural se analizó a través de SMARTPLS3.0

Tabla 1: Origen y definición de las escalas de medición

\begin{tabular}{|l|l|l|}
\hline \multicolumn{1}{|c|}{ Constructo } & \multicolumn{1}{|c|}{ Descripción } & \multicolumn{1}{c|}{ Autores } \\
\hline Imagen de la Universidad & $\begin{array}{l}\text { Percepción de Imagen que tiene la universidad en } \\
\text { su círculo, en los empleadores y público en general }\end{array}$ & $\begin{array}{l}\text { Fombrum, 1996; Chun, 2005; } \\
\text { Helgesen y Nesset, 2007. }\end{array}$ \\
\hline Percepción de Valor & $\begin{array}{l}\text { Percepción de los estudiantes respecto de relación } \\
\text { precio/calidad, costo/ganancia y del aporte de valor }\end{array}$ & Babin et al, 2005 \\
\hline Satisfacción & $\begin{array}{l}\text { Satisfacción que se reporta en comparación con } \\
\text { sus expectativas, con el ideal y universidad; Juicio } \\
\text { general y sumario de la satisfacción }\end{array}$ & Fornell, 1992; Ryan et al, 1995 \\
\hline Marketing Boca a Boca & $\begin{array}{l}\text { Intenciones de recomendación y comportamiento } \\
\text { de los estudiantes }\end{array}$ & Jalilvad y Samiei; 2012 \\
\hline Lealtad & $\begin{array}{l}\text { Juicio espontáneo de lealtad e intención de } \\
\text { proseguir estudios en la misma universidad }\end{array}$ & Yasvari, et al, 2012 \\
\hline
\end{tabular}

\section{RESULTADOS}

En primer lugar se analizó la fiabilidad y confiabilidad del modelo propuesto, según los indicadores mostrados en la tabla 2.

Para la fiabilidad individual de las variables asociadas al modelo se calcularon de los $\lambda$ (Loadings), 0 correlaciones simples de las medidas. Según afirma Carmines y Zeller (1979), para aceptar un indicador como integrante de un constructo este debe poseer una carga o $\lambda \geq 0,707$. Como se puede visualizar en la Tabla 2 todos los indicadores $\lambda$ cumplen con la condición de superar 0,707 . La fiabilidad del constructo fue medida utilizando el Alpha de Cronbach's, no obstante, se utilizó la Fiabilidad Compuesta (Composite Reliability), pues el Alpha de Cronbach's, tiende a subestimar la fiabilidad de la consistencia interna de las variables latentes (Bagozzi y Yi, 1988). Según Tabla 2, ambas medidas se encuentran sobre 0.7, lo que permite 
comprobar que existe fiabilidad de los constructos. La validez convergente del constructo se valoró a través de la varianza media extraída (AVE), el cual mide que la varianza del constructo se pueda explicar a través de los indicadores elegidos (Fornell y Larcker, 1981). Si este valor se encuentra sobre 0.5 indica que el constructo explica más de la mitad de la varianza de sus indicadores (Bagozzi y Yi, 1988). Los valores obtenidos nos permiten afirmar que existe validez convergente.

Respecto a la validez discriminante se evaluó utilizando el criterio de (Fornell y Larcker, 1981), el cual dice que la raíz cuadrada de AVE para cada constructo debe ser mayor a todas las correlaciones que éste tiene con el resto de constructos. Por otra parte, Bagozzi (1994), señala que las correlaciones entre los distintos factores que componen el modelo no deben ser superiores a 0.8 . Se puede verificar que, para el presente estudio, esto se cumple para cada uno de los constructos, por lo que se confirma la validez discriminante del modelo (ver Tabla 3). Como consecuencia de que todas las pruebas realizadas anteriormente han resultado positivas, se puede afirmar que el modelo de medida utilizado resulta válido y fiable.

Tabla 2: Indicadores de Fiabilidad y Confianza del Modelo Propuesto

\begin{tabular}{|c|c|c|c|c|c|}
\hline Dimensión & Item & Loadings $(\lambda)$ & $\begin{array}{l}\text { Alpha de } \\
\text { Cronbach's }\end{array}$ & $\begin{array}{l}\text { Composite } \\
\text { Reliability }\end{array}$ & $A V E$ \\
\hline \multirow[t]{5}{*}{ Satisfacción } & SAT1 & 0.897 & \multirow{5}{*}{0.939} & \multirow{5}{*}{0.953} & \multirow{5}{*}{0.803} \\
\hline & SAT2 & 0.894 & & & \\
\hline & SAT3 & 0.917 & & & \\
\hline & SAT4 & 0.893 & & & \\
\hline & SAT5 & 0.880 & & & \\
\hline \multirow{3}{*}{$\begin{array}{l}\text { Imagen de } \\
\text { la Universidad }\end{array}$} & IMG1 & 0.911 & \multirow{3}{*}{0.889} & \multirow{3}{*}{0.931} & \multirow{3}{*}{0.818} \\
\hline & IMG2 & 0.894 & & & \\
\hline & IMG3 & 0.908 & & & \\
\hline \multirow[t]{3}{*}{ Valor Percibido } & PERCVAL1 & 0.908 & \multirow{3}{*}{0.909} & \multirow{3}{*}{0.943} & \multirow{3}{*}{0.846} \\
\hline & PERCVAL2 & 0.941 & & & \\
\hline & PERCVAL3 & 0.910 & & & \\
\hline \multirow[t]{5}{*}{ Marketing Boca a Boca } & WOM1 & 0.878 & \multirow{5}{*}{0.965} & \multirow{5}{*}{0.873} & \multirow{5}{*}{0.877} \\
\hline & WOM2 & 0.953 & & & \\
\hline & WOM3 & 0.946 & & & \\
\hline & WOM4 & 0.962 & & & \\
\hline & WOM5 & 0.940 & & & \\
\hline \multirow[t]{3}{*}{ Lealtad } & LEAL1 & 0.909 & \multirow[b]{3}{*}{0.709} & \multirow[b]{3}{*}{0.835} & \multirow[b]{3}{*}{0.636} \\
\hline & LEAL2 & 0.868 & & & \\
\hline & LEAL3 & 0.572 & & & \\
\hline
\end{tabular}

Tabla 3: Matriz de Correlaciones y Validez Discriminante

\begin{tabular}{|l|c|c|c|c|c|}
\hline & $\begin{array}{c}\text { Imagen } \\
\text { Universidad }\end{array}$ & $\begin{array}{c}\text { Marketing } \\
\text { Boca a Boca }\end{array}$ & Lealtad & Satisfacción & $\begin{array}{c}\text { Valor } \\
\text { Percibido }\end{array}$ \\
\hline Imagen Universidad & $\underline{0.904}$ & & & & \\
\hline Marketing Boca a Boca & 0.617 & $\underline{0.936}$ & & & \\
\hline Lealtad & 0.528 & 0.703 & $\underline{0.797}$ & & \\
\hline Satisfacción & 0.608 & 0.794 & 0.669 & $\underline{0.896}$ & \\
\hline Valor Percibido & 0.619 & 0.730 & 0.588 & 0.721 & $\underline{0.920}$ \\
\hline \multicolumn{2}{|l|}{ En la diagonal, la raíz cuadrada del AVE } & & & \\
\hline
\end{tabular}

Con respecto al modelo estructural y la contrastación de hipótesis, se utilizó en primer lugar como criterio principal para la evaluación del modelo estructural es la cantidad de la varianza explicada $R^{2}$ (Chin, 1998). En la Tabla 4 , se puede apreciar los valores de $\mathrm{R}^{2}$ donde se muestra que el modelo explica en un $56,2 \%$ la Satisfacción, en un $44,7 \%$ la Lealtad y en un $68,4 \%$ el Marketing Boca a Boca. Por tanto, se puede señalar que el modelo sí refleja la Satisfacción, el Marketing Boca a Boca y la Lealtad, de los estudiantes de las Universidades de Consejo de Rectores de Chile estudiadas. Además, para cada relación o path entre 
constructos, los valores deseables deberían estar por encima de 0,3 teniendo como un valor mínimo 0,2 (Chin, 1998) Tal como se puede observar en la figura 2, todos los paths o caminos, superan el valor mínimo establecido.

Tabla 4: Resumen de resultados de análisis de datos para contraste de la Hipótesis $\mathrm{R}^{2}$

\begin{tabular}{|l|l|r|}
\hline Constructo & Tabulación & Varianza Explicada $R^{2}$ \\
\hline Marketing Boca a Boca & WOM & 0.684 \\
\hline Lealtad & LEAL & 0.447 \\
\hline Satisfacción & SATIF & 0.562 \\
\hline
\end{tabular}

Por otra parte, se realizó una evaluación del modelo estructural en su capacidad para predecir, la cual se mide a través del test Stone-Geisser Q2 (Geisser, 1974; Stone, 1974). Si Q2 es mayor que 0 esto implica que el modelo tiene relevancia predictiva (Chin, 1998; Hair et al., 2014). Como se puede apreciar en la Tabla 5, todos los Q2 obtenidos tienen signo positivo y son mayores que, lo que confirma la relevancia predictiva de los constructos exógenos sobre los constructos endógenos. Es decir, que es posible predecir el comportamiento de los constructos endógenos en este caso Satisfacción, Marketing Boca a Boca y Lealtad, a partir de los constructos exógenos.

Tabla 5: Resumen de resultados de análisis de datos para $\mathrm{Q}^{2}$

\begin{tabular}{|l|l|c|}
\hline Constructo & Tabulación & $Q^{2}$ \\
\hline Marketing Boca a Boca & WOM & 0.559 \\
\hline Lealtad & LEAL & 0.268 \\
\hline Satisfacción & SATIF & 0.422 \\
\hline
\end{tabular}

En la tabla 6 se muestra un resumen de los resultados del análisis de datos para el contraste de las hipótesis planteadas. Estos datos se ilustran en la figura 2. La medida en que las variables independientes contribuyen a la varianza explicada de las variables dependientes es recogida en el coeficiente $\beta$. Y para analizar la significancia de los coeficientes $\beta$ se utilizó el coeficiente t calculado mediante Bootstraping. El procedimiento de bootstrap fue realizado utilizando 5000 muestras de 317 casos y la opción "individual sign change" (Hair et al., 2011). El valor del coeficiente t obtenido se compara con la distribución t student con k-1 grados de libertad donde $\mathrm{k}$ es igual al número de muestras utilizadas. (Si el valor t se encuentra entre 1,65 y 1,95 el nivel de significancia es del $10 \%$; entre 1,96 y 2,157 el nivel es de $5 \%$ y si es superior a 2,58 el nivel de significancia es de $1 \%)$.

Tabla 6: Resumen de resultados de análisis de datos para contraste de la hipótesis planteadas (* Nivel de Significatividad $p<0,001$ y $\quad$ ** Nivel de Significatividad $p<0,010)$

\begin{tabular}{|c|l|l|l|l|l|l|}
\hline Hipótesis & \multicolumn{1}{|c|}{ Variable Independiente } & \multicolumn{1}{|c|}{ Variable Dependiente } & Beta & T-Statistic & $p$ & Resultado \\
\hline Hipótesis 1 & Imagen de la Universidad & Satisfacción & 0.261 & 4.909 & 0.000 & Soportada* $^{*}$ \\
\hline Hipótesis 2 & Valor Percibido & Satisfacción & 0.560 & 12.973 & 0.000 & Soportada* $^{*}$ \\
\hline Hipótesis 3 & Satisfacción & Lealtad & 0.669 & 19.308 & 0.000 & Soportada* $^{*}$ \\
\hline Hipótesis 4 & Satisfacción & Marketing Boca a Boca & 0.586 & 11.895 & 0.000 & Soportada $^{*}$ \\
\hline Hipótesis 5 & Lealtad & Marketing. Boca a Boca & 0.311 & 5.836 & 0.000 & Soportada $^{*}$ \\
\hline
\end{tabular}

Respecto de las hipótesis planteadas nos encontramos que quedan de la siguiente forma: La hipótesis 1 que planteaba que la Imagen de la Universidad influye directa y positivamente en la Satisfacción de sus estudiantes queda soportada con un Beta $=0.261$ y una significancia de $P=0.000$. La hipótesis 2 que proponía que el Valor Percibido tiene un impacto directo y positivo sobre la Satisfacción de los Estudiantes se soporta con un $B e t a=0.560$ y una significancia de $P=0.000$. La hipótesis 3 que proponía la relación directa entre la Satisfacción y la Lealtad de los estudiantes al igual que las hipótesis anteriores logra ser Soportada con un Beta de 0.669 y un nivel de significancia $P=0.000$. La hipótesis 4 que señalaba que la Satisfacción tiene una relación directa y positiva con la Intención de Marketing Boca a Boca, queda soportada con un Beta $=0.586$ y un nivel de significancia de $P=0.000$. Por último, se puede señalar que la hipótesis 5 que planteaba que la Lealtad estaba directa y positivamente relacionada con el Marketing Boca a Boca de los estudiantes también es soportada con un Beta $=0.311$ y una significancia de $P=0.000$. 


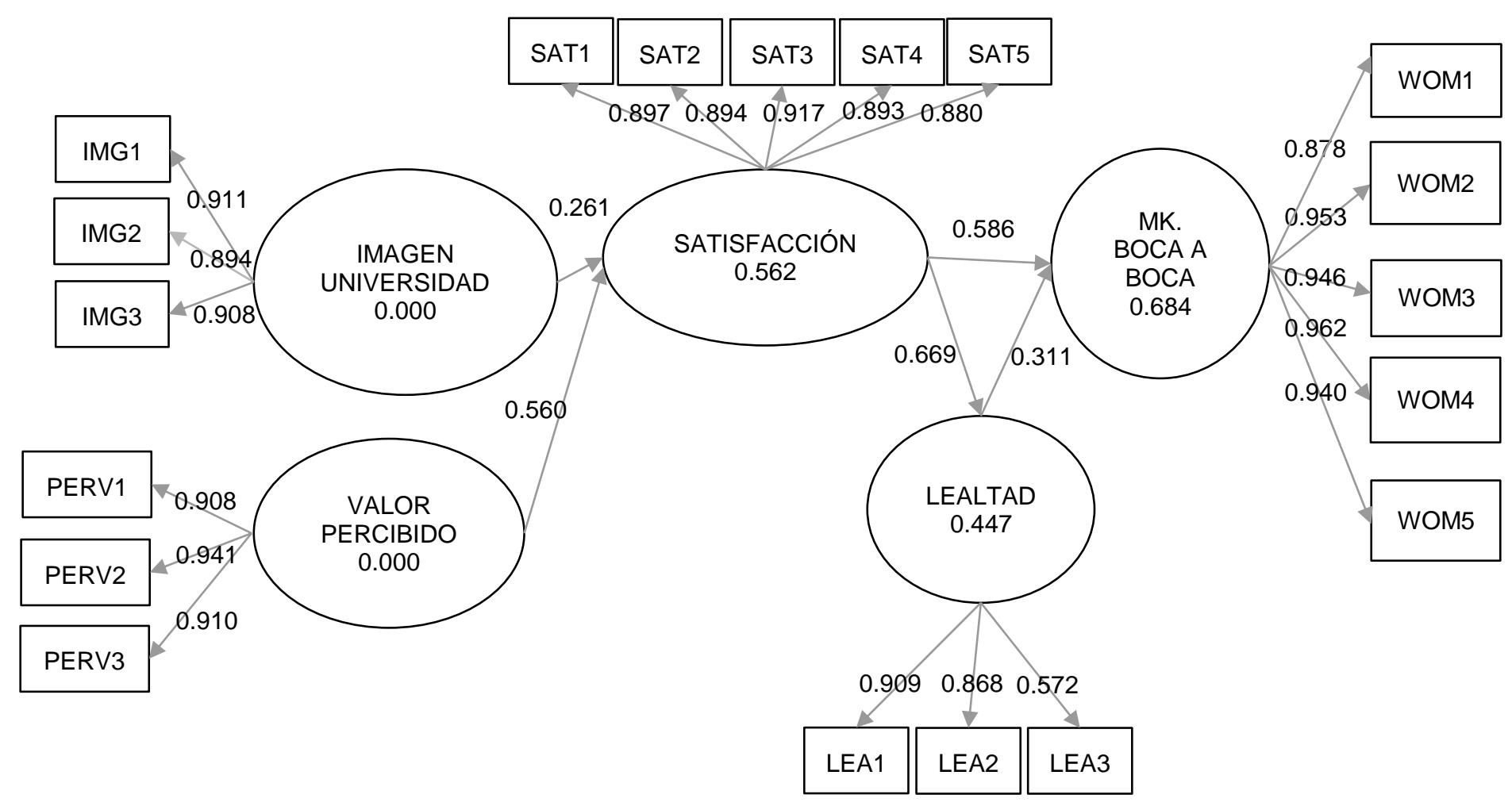

Fig. 2: Modelo Estructural con coeficientes path y $\mathrm{R}^{2}$

\section{CONCLUSIONES}

El objetivo principal de investigación fue examinar los factores que resultan eficaces en la formación de Marketing Boca a Boca acerca de las Universidades del Consejo de Rectores de Chile en particular de la ciudad de Antofagasta. El estudio permite confirmar las relaciones de causalidad entre las variables que integran el modelo estructural propuesto (Ver figura 2).

Los resultados obtenidos en el estudio muestran que la Percepción de Valor de los estudiantes respecto del servicio que le entrega su universidad resulta ser un claro antecedente de la Satisfacción de los estudiantes, tal como fue anteriormente señalado en los estudios de (Hartman y Schmidt ,1995; Webb y Jagun, 1997; Alves y Raposo, 2007). Por tanto, este resultado permite concluir quienes están encargados de la experiencia que vive el estudiante en su universidad, deben tener en cuenta y tener una preocupación especial por que exista un equilibrio entre los beneficios que la universidad es capaz de traspasar a sus estudiantes y los sacrificios que ellos realizan para la recepción del servicio.

Además, la imagen de la universidad resulta ser un antecedente de la satisfacción de los estudiantes en el contexto de la educación superior, en línea con previos estudios (Beerli et al., 2002; Schlesinger et al., 2017). Por otra parte, los resultados soportan que la satisfacción resultan ser un antecedente claro en el Marketing Boca a Boca, dicho resultado es consistente con los estudios previos acerca los efectos que presenta la satisfacción (Marzo et al., 2005b; De Matos y Rossi, 2008)

Resulta importante destacar también, que con este estudio se logra sustentar lo señalado por (Alves y Raposo, 2010; Schlesinger et al., 2014, Cabana et al., 2016), respecto del antecedente que resulta ser la Satisfacción para la Lealtad de los alumnos. Por tanto, mientras más satisfechos se encuentren los alumnos respecto de sus universidades, mayor propensión tendrán a continuar sus estudios en la misma Universidad hasta terminar sus carreras y además de volver a elegir su misma universidad en el caso de querer proseguir estudios de postgrado.

Por último, se establece que la Lealtad resulta ser un antecedente del Marketing boca a boca corroborando lo señalado por Chen (2016), respecto de que los alumnos con una mayor lealtad son quienes tienden a tener mayores conductas o intenciones asociadas a la generación de Marketing boca a boca.

Concluyendo, el presente estudio, buscaba profundizar sobre ciertos antecedentes del Marketing Boca a Boca en el contexto universitario. Se buscó contextualizar el mismo en los estudiantes de las universidades del 
consejo de rectores de la ciudad de Antofagasta. Sobre la base de los fundamentos teóricos y empíricos que fueron validados en el estudio se espera que tanto directivos, como rectores y resto de entidades implicadas en la estrategia de estas instituciones puedan trabajar en el diseño y desarrollo de nuevas políticas de marketing y gestión que apunten a la utilización del Marketing Boca a Boca como un elemento estratégico de bajo costo. El Marketing Boca a Boca puede permitir la mejora de las tasas de admisión de estudiantes universitarios, esto es la captación de más y mejores alumnos y, la retención de estos alumnos. Algunas medidas concretas que se derivan de este estudio sería la realización de estudios cualitativos acerca de los factores que inciden especialmente sobre la satisfacción de los estudiantes para destacar estos factores desde la gestión. También sería interesante mantener el contacto con los estudiantes egresados, por ejemplo para enviarles información de cómo proseguir estudios de postgrado. Ambas medidas podrán fomentar el Marketing Boca a Boca y, por ende, mejorar la captación de estudiantes.

\section{REFERENCIAS}

Alves, H. y M. Raposo, Conceptual model of student satisfaction in higher education, Total Quality Management, 18(5), 571-588 (2007)

Alves, H. y M. Raposo, The influence of university image on student behavior, International Journal of Educational Management, 24(1), 73-85 (2010)

Alves, H., The measurement of perceived value in higher education: a unidimensional approach, The Service Industries Journal, 31(12), 1943-1960 (2011)

Babin, B.J., Y. K. Lee, E. J. Kim y M. Griffin, Modeling consumer satisfaction and word-of-mouth: restaurant patronage in Korea, Journal of Services Marketing, 19(3), 133-139 (2005)

Bagozzi, R. P. e Y. Yi, On the evaluation of structural equation models, Journal of the academy of marketing science, 16(1), 74-94 (1988)

Bagozzi, R., The Effects of Arousal on the Organization of Positive and Negative Affect and Cognitions: Application to Attitude Theory, Structural Equation Modeling: A Multidisciplinary Journal, 1(3), 222-252 (1994)

Beerli Palacio, A., G. Díaz Meneses y P.J. Pérez Pérez, The configuration of the university image and its relationship with the satisfaction of students, Journal of Educational administration, 40(5), 486-505 (2002)

Brown, T.J., T. E. Barry, P.A. Dacin y R.F Gunst, Spreading the word: Investigating antecedents of consumers' positive word-of-mouth intentions and behaviors in a retailing context, Journal of the Academy of Marketing Science, 33(2), 123$138(2005)$

Brown, J. J. y P.H. Reingen, Social ties and word-of-mouth referral behavior, Journal of Consumer research, 14(3), 350362 (1987)

Bruner, J., G. Elacqua y otros cinco autores, Guiar el Mercado. Informe sobre la Educación Superior en Chile. Universidad Adolfo Ibañez, Santiago, Chile (2005)

Cabana, S. R., F.G. Cortés, D.L. Vega y R.A. Cortés, Análisis de la Fidelización del Estudiante de Ingeniería con su Centro de Educación Superior: Desafíos de Gestión Educacional, Formación Universitaria, 9(6), 93-104 (2016)

Carmines, E. G. y R.A. Zeller, Reliability and Validity Assessment. Quantitative applications in the social sciences 17. Bd., Newbury Park, CA (1979)

Chen, C.T., The Investigation on Brand Image of University Education and Students' Word-of-Mouth Behavior, Higher Education Studies, 6(4), 23-33 (2016)

Chin, W.W., The Partial Least Squares Approach for Structural Equation Modeling, Modern Methods for Business Research, 295(2), 295-336 (1998)

Chun, R., Corporate Reputation: Meaning and Measurement, International J. of Management Reviews, 7(2), 91-109 (2005)

Díaz-Méndez, M. y E. Gummesson, Value co-creation and university teaching quality: Consequences for the European Higher Education Area (EHEA), Journal of Service Management, 23(4), 571-592 (2012)

De Matos, C. A. y C.A.V. Rossi, Word-of-mouth communications in marketing: a meta-analytic review of the antecedents and moderators, Journal of the Academy of Marketing Science, 36(4), 578-596 (2008)

Fielden, J. S., C.B. Hilton y W.H. Motes, Educational services marketing: a proposed system for enhanced recruitment of students, Journal of Professional Services Marketing, 8(2), 191-205 (1993)

Fombrun, C. J., Reputation: Realizing Value from Corporate Brand Management. Boston: Harvard Business School Pres, Boston (1996)

Fornell, C. y D.F. Larcker, Evaluating structural equation models with unobservable variables and measurement error, Journal of marketing research, 39-50 (1981)

Fornell, C., A national customer satisfaction barometer: The Swedish experience, Journal of Marketing, 56, 6-21 (1992)

Geisser, S., A predictive Approach to the Random Effect Model, Biometrika, 61(1), 101-107(1974) 
Hair, J.F., C.M. Ringle y M. Sarstedt, PLS-SEM: Indeed a silver bullet. Journal of Marketing theory and Practice, 19(2), 139-152(2011)

Hair, J.F., M. Sarstedt, L. Hopkins y V.G. Kuppelwieser, Partial least squares structural equation modeling (PLS-SEM): An emerging tool in business research, European Business Review, 26(2), 106-121(2014)

Hartman, D.E. y S.L. Schmidt, Understanding student/alumni satisfaction from a consumer's perspective: The effects of institutional performance and program outcomes, Research in Higher Education, 36(2), 197-217(1995)

Harrison-Walker, L.J., The measurement of word-of-mouth communication and an investigation of service quality and customer commitment as potential antecedents, Journal of service research, 4(1), 60-75 (2001)

Harrison-Walker, L.J., Customer prioritization in higher education: targeting 'right'students for long-term profitability, Journal of Marketing for Higher Education, 20(2), 191-208 (2010)

Heitmann, M., D.R. Lehmann y A. Herrmann, Choice goal attainment and decision and consumption satisfaction, Journal of marketing research, 44(2), 234-250 (2007)

Helgesen, $\varnothing$ y E. Nesset, Images, satisfaction and antecedents: Drivers of student loyalty? A case study of a Norwegian university college, Corporate Reputation Review, 10(1), 38-59 (2007)

Jagdish, S., D.M. Gardner y D.E. Garret, Marketing theory: evolution and evaluation, New York, Wiley (1994)

Jalilvand, M. y N. Samiei, The effect of electronic Word of Mouth on Brand Image and purchase intention: An empirical study in the automobile industry in Iran, Marketing Intelligence \& Planning, 30 (4), 460 - 476 (2012)

Kazoleas, D., Y. Kim y M. Anne Moffitt, Institutional image: a case study. Corporate Communications: an international journal, 6(4), 205-216 (2001)

Lam, S. Y., V.Shankar, K.M. Erramilli y B. Murthy, Customer value, satisfaction, loyalty, and switching costs: An illustration from a business-to-business service context, Journal of the academy of marketing science, 32(3), 293-311(2004)

Luque, T. y S. del Barrio, Análisis del valor de las percepciones de los clientes en el diagnóstico estratégico de la universidad. In Ponencia presentada en el International Congress Marketing Trends, 26-27 (2007)

Martín-Armario, E., M.C. Barroso-Castro y D. Martín-Ruiz, El valor percibido de un servicio, Revista española de investigación de marketing, ISSN, 1138-442 (2004)

Marzo-Navarro, M., M. Pedraja-Iglesias y M. Rivera-Torres, A new management element for universities: satisfaction with the offered courses, International Journal of educational management, 19(6), 505-526 (2005)

Marzo-Navarro, M., M. Pedraja-Iglesias y M. Rivera-Torres, Measuring customer satisfaction in summer courses, Quality Assurance in Education, 13(1), 53-65 (2005)

Maric, M., J. Pavlin y M. Ferjan, Educational Institution's image: A case study, Organizacija, 43, 58- 65 (2010)

Maxham III, J. G. y R. G. Netemeyer, A longitudinal study of complaining customers' evaluations of multiple service failures and recovery efforts, Journal of marketing, 66(4), 57-71 (2002)

Monroe, K. B., Pricing: Making Profitable Decisions, McGraw-Hill, New York (1990)

Mourali, M., M. Laroche y F. Pons, Antecedents of consumer relative preference for interpersonal information sources in pre-purchase search, Journal of Consumer Behaviour, 4(5), 307-318 (2005)

Oliver, R.L., A cognitive model of the antecedents and consequences of satisfaction decisions, Journal of marketing research, 460-469 (1980)

Oliver, R. L., Whence consumer loyalty? The Journal of Marketing, 33-44(1999)

Oliver, R. L. y W. S. DeSarbo, Response determinants in satisfaction judgments. Journal of consumer research, 14(4), 495-507 (1988)

Ranaweera, C. y J. Prabhu, On the relative importance of customer satisfaction and trust as determinants of customer retention and positive word of mouth, Journal of Targeting, Measurement and Analysis for marketing, 12(1), 82-90 (2003)

Ryan, M.J., T. Buzas y V. Ramaswamy, Making CSM a power tool: Composite indices boost the value of satisfaction measures for decision making, Marketing Research, 7(3), 11-16 (1995)

Rogers, J., y M. Smith, Demonstrating genuine interest in students' needs and progress: Implications for student satisfaction with courses, Journal of Applied Research in Higher Education, 3(1), 6-14 (2011)

Sanzo, M.J., M.L. Santos, R. Vázquez y L.I. Álvarez, The effect of market orientation on buyer-seller relationship satisfaction. Industrial Marketing Management, 32(4), 327-345 (2003)

Schlesinger, W., A. Cervera y H. Calderón. El papel de la confianza, la imagen y los valores compartidos en la creación de valor y lealtad: aplicación a la relación egresado-universidad, Revista Española de Investigación en Marketing ESIC, 18(2), 126-139 (2014)

Schlesinger, W., A. Cervera y C. Pérez-Cabañero, Sticking with your university: the importance of satisfaction, trust, image, and shared values, Studies in Higher Education, 42(12), 2178-2194 (2017) 
Schertzer, C. B. y S. M. Schertzer, Student satisfaction and retention: A conceptual model, Journal of Marketing for Higher Education, 14(1), 79-91 (2004)

Selnes, F., Antecedents and consequences of trust and satisfaction in buyer-seller relationships, European Journal of marketing, 32(3/4), 305-322 (1998)

Silverman, G., The Secrets of Word-of-Mouth Marketing: How to Trigger Exponential Sales through Runaway Word of Mouth. AMACOM Div American Mgmt Assn.New York, NY (2001)

Stone, M., Cross-validatory choice and assessment of statistical predictions, Journal of the royal statistical society, Series B (Methodological), 111-147 (1974)

Sweeney, J.C., G.N. Soutar y T. Mazzarol, Factors influencing word of mouth effectiveness: receiver perspectives, European Journal of Marketing, 42(3/4), 344-364 (2008)

Wangenheim, F.V. y T. Bayón, The chain from customer satisfaction via word-of-mouth referrals to new customer acquisition, Journal of the Academy of Marketing Science, 35(2), 233-249 (2007)

Webb, D. y A. Jagun. Customer care, customer satisfaction, value, loyalty and complaining behavior: validation in a UK university setting, Journal of Consumer Satisfaction, Dissatisfaction and Complaining Behavior, 10 (1997)

Yasvari, T. H., R. A. Ghassemi y E. Rahrovy, Influential Factors on Word of Mouth in Service Industries (The case of Iran Airline Company), International Journal of Learning and Development, 2(5), 227-242 (2012)

Zeithaml, V. A., Consumer perceptions of price, quality, and value: a means-end model and synthesis of evidence, The Journal of marketing, 2-22 (1988) 